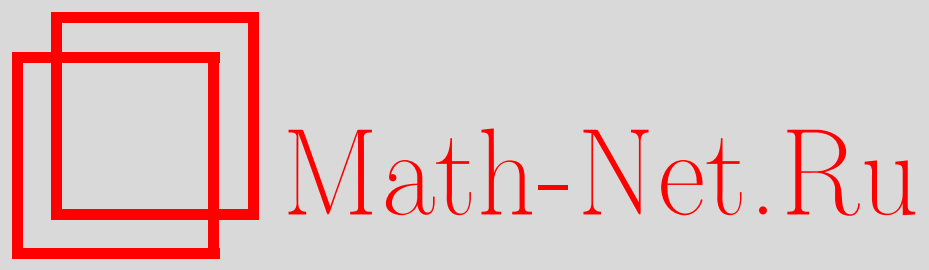

Г. М. Губреев, В. Н. Пивоварчик, Спектральный анализ задачи Редже с параметрами, Функи. анализ и его прил., 1997, том 31, выпуск 1, 70-74

DOI: https://doi.org/10.4213/faa447

Использование Общероссийского математического портала MathNet.Ru подразумевает, что вы прочитали и согласны с пользовательским соглашением

http://www . mathnet.ru/rus/agreement

Параметры загрузки:

IP : 3.85 .5 .30

26 апреля 2023 г., 15:54:16

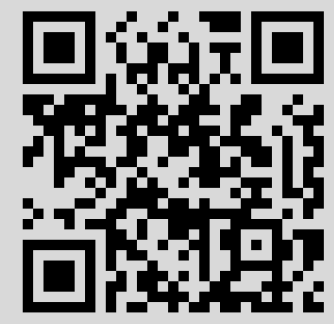


Наконец, заметим, что к доказательству неравенств $\mu_{k}(T)<\nu_{k+1}(T)$ сводится строгое обоснование результатов об отсутствии рассеяния назад краевых состояний в квантовом эФФекте Холла [6]; в ряде работ эти результаты получались численными методами.

В заключение авторы благодарят рецензента за замечания, способствовавшие улучшению рукописи.

\title{
ЛитеРАтУРА
}

1. Рид М., Саймон Б. Методы современной математической физики, т. 4. Мир, М., 1982 . 2. Станкевич И. В. ДАН СССР, 192, №1, 34-37 (1970). 3. Марченко В. А., Островский И. В. Матем. сб., 97, вып. 4, 540-606 (1975). 4. Garnett J., Trubowitz E. Comment. Math. Helv., 59, 258-315 (1984). 5. Левитан Б. М., Сарәсян И. С. Операторы Штурма-Лиувилля и Дирака. Наука, М., 1988. 6. Квантовый эффект Холла. Сб. статей (Сост. А. Я. Шик, Ю. В. Шмарцев), Мир, М., 1986.

Мордовский государственный университет им. Н. П. Огарёва
Поступило в редакцию 20 февраля 1996 г.

\section{Спектральный анализ задачи Редже с параметрами}

\author{
(c) 1997. Г. М. ГуБРеЕв, В. Н. ПивовАРЧИк
}

Заметка посвящена исследованию спектральной задачи

$$
\begin{gathered}
-y^{\prime \prime}+q(x) y=\lambda^{2} y, \\
y(0)=0, \quad y^{\prime}(a)+(i \alpha \lambda+\beta) y(a)=0, \quad \alpha>0, \beta \in \mathbb{R},
\end{gathered}
$$

с вещественным потенциалом $q \in L_{2}(0, a)$ и параметрами $\alpha, \beta$. Введение параметров объясняется тем, что к такой задаче с помощью преобразования Лиувилля приводится задача о колебаниях гладкой струны с вязким трением на правом конще, причем ограничение $\alpha>0$ вызвано тем, что этот параметр пропорционален коэффициенту трения. При $\alpha=1, \beta=0$ получаем классическую задачу Редже [1], возникающую при описании рассеяния на финитном потенциале. В [1] для случая однократного спектра доказана полнота системы собственных функций задачи в пространстве $L_{2}(0,2 a)$ в предположении, что $a \in \operatorname{supp} q$. Отсюда выводится (кстати, некорректно) возможность разложения в ряды по собственным функциям задачи произвольной функции из $L_{2}(0,2 a)$. Опираясь на эти разложения, автор дает решение интегрального уравнения ГельфандаЛевитана, которое позволяет восстановить потенциал по заданной матрице рассеяния.

Настоящая заметка посвящена развитию этих исследований. Оставляя в стороне общий случай, объясним суть этого развития на примере задачи Редже $(\alpha=1, \beta=0)$. Здесь впервые дано полное описание спектра такой задачи

* Эта работа выполнена при поддержке Международного научного фонда, гранты $\mathrm{UCZ000} \mathrm{и} \mathrm{UCZ200.}$ 
и доказана теорема единственности (теоремы 1, 3). Это позволило, опираясь на процедуру Левитана-Гасымова-Марченко, дать решение обратной задачи для потенциалов класса $L_{2}(0, a)$ только по спектру (а не по матрице рассеяния). Далее, теорема Редже о полноте является частным случаем теоремы 5, в которой фактически определяется зависимость интервала полноты от расположения носителя потенциала $q$. Отметим также, что теорема 6 является новой и для случая классической задачи Редже.

1. Спектр и обратная задача. Спектр задачи (1)-(2) совпадает с множеством корней целой функции экспоненциального типа

$$
\varphi(\lambda)=s^{\prime}(\lambda, a)+(i \alpha \lambda+\beta) s(\lambda, a),
$$

где $s(\lambda, x)$ - решение уравнения (1), удовлетворяющее условиям $s(\lambda, 0)=$ $s^{\prime}(\lambda, 0)-1=0$. Спектр симметричен относительно мнимой оси и все достаточно большие по модулю собственные значения простые (за исключением, быть может, случая $\alpha=1, \beta=0)$. Симметричные относительно мнимой оси собственные значения имеют одинаковую кратность. В замкнутой нижней полуплоскости возможно лишь конечное число собственных значений. Они чисто мнимые и простые; их число не зависит от $\alpha>0$ и его можно оценить. Например, при $\beta=0$ число собственных значений в замкнутой нижней полуплоскости не превосходит величины $\int_{0}^{a} x|q(x)| d x$. Перечисленные результаты получены путем сведения задачи (1)-(2) к квадратичному операторному пучку специального вида [2].

Дадим теперь характеристику всех возможных спектров задач Редже (1)-(2) с потенциалами $q \in L_{2}(0, a)$. Возьмем две перемежающиеся последовательности вещественных чисел

$$
-\infty<\nu_{1}<\mu_{1}<\nu_{2}<\mu_{2}<\cdots
$$

с асимптотиками

$$
\nu_{k}=\frac{\pi^{2}}{a^{2}}\left(k-\frac{1}{2}\right)^{2}-2 p+\beta_{k}, \quad \mu_{k}=\frac{\pi^{2}}{a^{2}} k^{2}-2 p+\alpha_{k},
$$

где $p \in \mathbb{R},\left\{\alpha_{k}\right\}_{1}^{\infty},\left\{\beta_{k}\right\}_{1}^{\infty} \in l_{2}$, и введем в рассмотрение целые функции

$$
\theta_{1}(\lambda) \stackrel{\mathrm{df}}{=} \prod_{1}^{\infty}\left(\frac{\pi}{a}\left(k-\frac{1}{2}\right)\right)^{-2}\left(\nu_{k}-\lambda^{2}\right), \quad \theta_{2}(\lambda) \stackrel{\mathrm{df}}{=} a \prod_{1}^{\infty}\left(\frac{\pi}{a} k\right)^{-2}\left(\mu_{k}-\lambda^{2}\right) .
$$

Через $\mathfrak{R}_{a}$ обозначим множество функций вида

$$
\varphi(\lambda)=\theta_{1}(\lambda)+(i \alpha \lambda+\beta) \theta_{2}(\lambda), \quad \alpha>0, \beta \in \mathbb{R} .
$$

Теорема 1. Для того чтобы множество $\Lambda$ било спектром задачи Редже (1)-(2), необходимо и достаточно, чтобы оно совпадало с множеством корней некоторой функиии класса $\mathfrak{R}_{a}$.

В случае $\alpha \neq 1$ можно дать явное описание спектра задачи Редже. Обозначим через $Q_{\beta}$ множество потенциалов $q$, для которых оператор $(A y)(x)=-y^{\prime \prime}+$ $q(x) y$ с областью определения $D(A)=\left\{y \in W_{2}^{2}(0, a), y(0)=y^{\prime}(a)+\beta y(a)=0\right\}$ строго положителен. Именно такой класс потенциалов соответствует равномерно положительной плотности распределения массы в аналоге задачи Редже для струны. Например, в случае $\alpha \in(0,1)$ из теоремы 1 выводится теорема 2. 
Теорема 2. Для того чтобь последовательность $\Lambda=\left\{\lambda_{k}\right\}_{1}^{\infty} \cup\left\{\lambda_{k}\right\}_{-\infty}^{-1}$ была спектром задачи (1)-(2) с потенциалом $q \in Q_{\beta}$ при некоторых $\beta \in \mathbb{R}$ $u \quad \alpha \in(0,1)$, необходимо и достаточно выполнение следуюших условий: (i) $\operatorname{Im} \lambda_{k}>0, \lambda_{k} \in \Lambda$; (ii) число чисто мнимых $\lambda_{k}$ (с учетом кратностей) четно; (iii) $\Lambda$ симметрична относительно мнимой оси и кратности симметрично расположенных точек совпадают; (iv) при соответствующей нумерачии правая ветвь $\left\{\lambda_{k}\right\}_{1}^{\infty}$ удовлетворяет асимптотическим формулам

$$
\lambda_{n}=\frac{\pi}{a}\left(n-\frac{1}{2}\right)+i G+\frac{H}{n}+\frac{b_{n}}{n}, \quad G>0, H \in \mathbb{R},\left\{b_{n}\right\}_{1}^{\infty} \in l_{2} .
$$

Аналогичный результат с несколько измененными формулами (4) имеет место и в случае $\alpha>1$.

Перейдем теперь к процедуре восстановления параметров задачи по спектру. Через $S\{q, \alpha, \beta, a\}$ обозначим спектр задачи Редже на отрезке $[0, a]$ с $q \in L_{2}(0, a), \alpha>0, \beta \in \mathbb{R}$.

Tеорема 3. Если $S\left\{q_{1}, \alpha_{1}, \beta_{1}, a\right\}=S\left\{q_{2}, \alpha_{2}, \beta_{2}, a\right\}$, mo $q_{1}(x) \stackrel{\text { ח.в. }}{=} q_{2}(x)$, $\alpha_{1}=\alpha_{2}, \beta_{1}=\beta_{2}$.

Чтобы сформулировать следующий результат, введем такие обозначения. Если $\Lambda=\left\{\lambda_{k}\right\}_{-\infty}^{\infty}$ является спектром задачи $(1)-(2)$ на отрезке $[0, a]$, то полагаем

$$
\begin{gathered}
d=a-\pi \lim _{r \rightarrow \infty} \frac{n_{+}(r)}{r}, \quad n_{+}(r)=\operatorname{card}\left\{\lambda_{k} \in \Lambda:\left|\lambda_{k}\right|<r, \operatorname{Re} \lambda_{k}>0\right\}, \\
\varphi(\lambda)=C \psi(\lambda), \quad \psi(\lambda)=e^{i d \lambda} \text { v.p. } \prod_{\lambda_{k} \in \Lambda}\left(1-\frac{\lambda}{\lambda_{k}}\right),
\end{gathered}
$$

где константа $C$ однозначно определяется условием существования предела

$$
\lim _{\lambda \rightarrow \infty} \frac{C \psi(\lambda)-\cos \lambda a}{i \sin \lambda a},
$$

причем здесь, а также в формулах следующей ниже теоремы, $\lambda$ стремится к $\infty$, оставаясь на какой-нибудь прямой, параллельной вещественной оси. Формулы (5)-(6) корректны, поскольку $\Lambda$ является множеством корней функции класса М. Картрайт [3]. Далее, если $\Lambda=\varnothing$, то полагаем, что $\psi(\lambda)=\exp \{i a \lambda\}$. И наконец, введем обозначения

$$
\varphi_{\mathrm{H}}(\lambda)=\frac{1}{2}(\varphi(\lambda)-\varphi(-\lambda)), \quad \varphi_{\mathrm{Y}}=\frac{1}{2}(\varphi(\lambda)+\varphi(-\lambda)) .
$$

Teорема 4. Пусть $\Lambda$ - спектр задачи Редже (1)-(2) на $[0, a]$ с $q \in$ $L_{2}(0, a)$. Тогда параметры задачи восстанавливаются по формулам

$$
\alpha=\lim _{\lambda \rightarrow \infty} \frac{C \psi(\lambda)-\cos \lambda a}{i \sin \lambda a}, \quad \beta=i \alpha \lim _{\lambda \rightarrow \infty} \frac{\lambda \varphi_{\mathrm{Y}}(\lambda)-\lambda \cos \lambda a-\gamma \sin \lambda a}{\varphi_{\mathrm{H}}(\lambda)},
$$

где $\gamma=2(i \alpha \pi)^{-1} \int_{0}^{\infty}\left(\varphi_{\mathrm{H}}(\lambda)-i \alpha \sin \lambda a\right) \sin \lambda a d \lambda$. Далее, квадратьи корней функиий

$$
\theta_{1}(\lambda) \stackrel{\text { df }}{=} \varphi_{\mathrm{Y}}(\lambda)-\beta(i \alpha)^{-1} \varphi_{\mathrm{H}}(\lambda) \lambda^{-1}, \quad \theta_{2}(\lambda) \stackrel{\text { df }}{=} \varphi_{\mathrm{H}}(\lambda) \lambda^{-1}
$$


задают два спектра краевых задач для уравнения (1) с граничньли условиями $y(0)=y(a)=0, y(0)=y^{\prime}(a)=0$, по которым однозначно определяется $q \in L_{2}(0, a)$ в силу канонической прочедурь [4].

СледствиЕ. (а) Спектр задачи Редже пуст в том и только том случае, когда $q(x) \stackrel{\text { п.в. }}{=} 0, \alpha=1, \beta=0$.

(b) Cпектр не может бить конечным множеством.

2. Полнота и базисность. Снова рассмотрим задачу (1)-(2), предполагая на этот раз, что $q$ задан при всех $x \geqslant 0$ и $q \in L_{2}^{\text {loc }}\left(\mathbb{R}_{+}\right)$. Спектр такой задачи снова совпадает с множеством корней функции (3), однако теперь соответствующие собственные и присоединенные функции определены при всех $x \in \mathbb{R}$. В этом пункте мы вычислим такое число $T$, что система корневых функций задачи Редже полна и минимальна в пространстве $L_{2}(0, T)$, а также обсудим вопрос о базисности этой системы. Такая постановка инициирована работой [5], в которой рассмотрены аналогичные задачи для случая струны. Отметим, что доказательства теорем 5, 6 полностью основаны на результатах работы [6]. Положим

$$
T=h_{\varphi}(\pi / 2)+h_{\varphi}(-\pi / 2)
$$

где $h_{\varphi}$ - индикатор роста функции $\varphi$, определяемой равенством $(3)$. Из $(5),(6)$ следует и другое выражение для $T$ :

$$
T=2 \pi \lim _{r \rightarrow \infty} r^{-1} n_{+}(r) .
$$

Теорема 5. Система корневых функиий задачи Редже с параметрами $\alpha>0, \beta$ полна в пространстве $L_{2}(0, b)$, если $b<T$, полна и минимальна, если $b=T$, и не полна, если $b>T$.

Из $(7)$ вытекает, что $0 \leqslant T \leqslant 2 a$, причем равенство $T=2 a$ имеет место, например, в случае $\alpha \neq 1$ (но не только в этом случае). С другой стороны, $T$ может быть сколь угодно малым и $T=0$, если спектр задачи пуст. Это объясняется тем, что при $\alpha=1, \beta=0$ имеет место оценка $T \leqslant 2 b$, где $b$ крайняя правая точка множества $\operatorname{supp} q$ на сегменте $[0, a]$.

Теорема 6. (i) Если $\alpha \neq 1$, то при любом $\beta \in \mathbb{R}$ система корневьх функиий задачи Редже образует безусловный базис пространства $L_{2}(0, T)$.

(ii) Если $\alpha=1, \beta \neq 0$, то система корневых функций задачи Редже не образует безусловного базиса пространства $L_{2}(0, T)$.

(iii) Если $\alpha=1, \beta=0$, а потенциал таков, что при некотором $\nu>0$

$$
q(x) \sim C(x-a)^{\nu}, \quad x \rightarrow a-0, C \neq 0,
$$

то система корневых функций не образует безусловного базиса пространства $L_{2}(0, T)$.

Объясним вкратце, чем вызвана небазисность системы корневых функций в двух последних случаях. Дело в том, что в этих случаях мнимые части собственных значений $\lambda_{n}$ растут как $\log |n|$ и, стало быть, последовательность $\Lambda$ не удовлетворяет условию Карлесона. С другой стороны, доказывается, что карлесоновость спектра необходима для базисности.

Авторы выражают признательность участникам семинара Д. З. Арова за обсуждение результатов. 


\title{
ЛитеРАтУРА
}

1. Regge T. Nuovo Cimento, 9, No. 3. 491-503 (1958). 2. Баркарь С. М., Пивоварчик В. Н. Изв. Акад наук респ. Молдова, 1 (7), 17-28 (1992). 3. Левин Б. Я. Распределение корней целых функций. ГТИЗ, М., 1956. 4. Марченко В. А. Операторы Штурма-Лиувилля и их приложения. Наукова думка, Киев, 1977. 5. Hrusev S. V., Nikolskii N. K., Pavlov B. S. Lect. Notes in Math, Vol. 864 (1981), pp. 214-335. 6. Гyбреев Г. М. Записки научн. сем. ЛОМИ. Исслед. по теор. опер. и теор. функ., 19, 34-80 (1991).

Южно-Украинский педагогический университет Одесская государственная Академия

Поступило в редакцию строительства и архитектуры

30 января 1996 г.

\section{О существовании деформаций без критических точек (задача Тесье для функций двух переменных) ${ }^{\star}$}

\author{
(C) 1997. С. М. ГУСЕЙН-ЗАДЕ
}

Пусть $f:\left(\mathbb{R}^{n}, 0\right) \rightarrow(\mathbb{R}, 0)$ - росток $C^{\infty}$-функции с алгебраически изолированной критической точкой в начале координат, $f$ - представитель ростка $f$, определенный в окрестности $U$ начала координат, $f_{\lambda}-$ семейство функций на $U$, гладкое по $\lambda$ из окрестности нуля в $\mathbb{R}$ и такое, что $f_{0}=f$. Функция $\tilde{f}=f_{\lambda}$ для достаточно малых $\lambda \neq 0$ будет называться (малой) деформацией ростка $f$.

Для достаточно малых $\delta$ и $\varepsilon$, таких, что $0<|\varepsilon| \ll \delta$, пусть $M_{\varepsilon}=M_{\varepsilon}(f)=$ $\left\{z \in \mathbb{R}^{n},\|z\| \leqslant \delta: f(z) \leqslant \varepsilon\right\}$ - множество меньших значений ростка $f$. Для $\varepsilon>0$ пространство $M_{+\varepsilon}$ стягиваемо, а пространство $M_{-\varepsilon}$ гомотопически эквивалентно многообразию уровня $V_{-\varepsilon}=f^{-1}(-\varepsilon) \cap B_{\delta}$. Существуют очевидные топологические препятствия к существованию деформаций ростка $f$ без критических точек. Если пара $\left(M_{+\varepsilon}, M_{-\varepsilon}\right)(0<\varepsilon \ll \delta)$ гомотопически нетривиальна (т. е. если $M_{-\varepsilon}$ не является деформационным ретрактом множества $M_{+\varepsilon}$ ), то любая малая деформация ростка $f$ имеет критическую точку в $B_{\delta}$. Пара $\left(M_{+\varepsilon}, M_{-\varepsilon}\right)$ гомотопически тривиальна тогда и только тогда, когда пространство $M_{-\varepsilon}$ стягиваемо. Варианты следующего вопроса известны под названием, упомянутым в заголовке.

Верно ли, что если пространство $M_{-\varepsilon}$ стяаиваемо, то росток $f$ имеет деформачию без критических точек?

Мы даем ответ на этот вопрос для функций двух переменных. Пусть $f:\left(\mathbb{R}^{2}, 0\right) \rightarrow(\mathbb{R}, 0)$ - росток функции двух переменных. Если множество $\{f=0\} \cap B_{\delta}$ не состоит из одной точки, то $M_{-\varepsilon}$ гомотопически эквивалентно несвязному объединению $r$ точек, где $r$ - количество ветвей кривой $\{f=0\}$. Поэтому $M_{-\varepsilon}$ стягиваемо тогда и только тогда, когда кривая $\{f=0\}$ имеет одну вещественную ветвь. Далее мы будем считать, что функция $f$ определена 